\title{
Multi-State Diagnosis and Prognosis of Lubricating Oil Degradation using Sticky Hierarchical Dirichlet Process -Hidden Markov Model Framework
}

Monika Tanwar ${ }^{1}$, Hyunseok Park ${ }^{2}$, and Nagarajan Raghavan ${ }^{1}$

${ }^{1}$ Engineering Product and Development Pillar, Singapore University of Technology and Design

${ }^{2}$ Department of Information Systems, College of Engineering, Hanyang University

May 7, 2021

\section{Hosted file}

HDP-HMM Manuscript.pdf available at https://authorea.com/users/412378/articles/521082-multistate-diagnosis-and-prognosis-of-lubricating-oil-degradation-using-sticky-hierarchicaldirichlet-process-hidden-markov-model-framework 